\title{
Correlation between Sympathtic Power and Left Ventricular Ejection Fraction in Diabetics and Hypertensives
}

\section{Manjusha Joshi ${ }^{1 *}$, Desai $\mathrm{KD}^{2}$ and Menon $\mathrm{MS}^{3}$}

${ }^{1}$ Electronics and Telecommunication Department, MPSTME /NMIMS Deemed to be University, Bhakti Vedant Marg, Vile parle-w, Mumbai, India ${ }^{2}$ Maharshi Parashuram College of Engineering, Mumbai University, India

${ }^{3}$ Cardiology Department, Fortis-S.L. Raheja Hospital, Mahim-W, Mumbai, India

\begin{abstract}
Cardiac diseases are more prevalent in diabetics, hypertensives and diabetics with hypertentension. Prevention is possible if they are diagnosed at the preclinical stage. Such diagnostic practises can control mrotality and morbidity rate.

Methods and subjects: Study is conducted with 27 normal subjects, 40 diabetic subjects with and without myocardial ischemia/infaction and 23 hypertensive subjects with and without diabetes. An attempt is made to find out the sympathetic power and it's correlation to Left Ventricular Ejection Fraction in the diabetic subjects and hypertensive subjects with and without diabetes. All the cases are recorded at Fortis-S.L.Raheja hospital Mahim (W). ECG samples of 3-5 minute duration in sittng and supine position are collected. Randomness in age, class, sex and other parameters in ensured on the basis of the data collection as per the registration of the subjects.
\end{abstract}

Keywords: II lead ECG; Heart rate variability; Sympathetic power; Auto regressive analysis; Glucose metabolism; Atheroscelerosis; Dislipidemia and left ventricular ejection fraction

\section{Introduction}

Third world countries are largest contributors for global deaths due to cardiac diseases [1]. The effects of unhealthy diet and physical inactivity may show up in individuals as raised blood pressure, raised blood glucose, raised blood lipids, overweight and obesity. High carbohydratic and high fat diet and physical inactivity are the major contributors to abnormal lifestyle diseases like diabetes and hypertension [2]. Under prevailing diabetes or hypertension and combined effect of both, gradual deterioration in cardiac performance is commonly observed. This deterioration culminates into a cardiac stroke. The gradual deterioration of cardiac performance is visible in only in heart rate variability (HRV) analysis at an early stage where no clinical symptoms of cardiac functional deterioration are visible. The HRV analysis is not used in current clinical practice. The attempt to establish correlation between the echocardiogram findings and HRV analysis is focused towards validating the HRV analysis as an early diagnostic tool that can be used to control the morbidity and mortality rate due to cardiac diseases [3].

\section{Overview of diabetes, hypertension and associated complications}

Diabetes causes multiple disorders where the glucose metabolism is changed to protein and fat metabolism. Almost all body organs are deprived of the nutrients due to protien and fat metabolism [4]. Protein and fat metabolism cause atheroscelerosis and dislipidemia [3]. Vasculature is affected due to long term hypoglycemia $[3,4]$. Reduced signal conduction is observed under microvascular and microvasculature. The domination of parasympathetic hormone is observed in diabetic subjects. In diabetic subjects, the heart cannot fulfill the sudden demand of blood required to carry out different daily activities due to domination of parasympathetic hormone. As a result, diabetic subjects experience morbidity. Occlusion in blood vassels is more prevalent. If the coronary artery is occluded, the cardiac disease is resulted. The prevailing diabetic condition with prevalence of atherosclerosis causes hypertension. Under prevaling diabetes, along with many other complications, the risk factor of cardiac morbidity and mortality increases.

Hypertension is either essential or systemic hypertension or secondary hypertension. Essential hypertension is caused due to hereditory traits, sedentary lifestyle or stiffening of blood vessels due to aging. The prevalence of such hypertension is $90 \%-95 \%$. Secondary hypertension is caused due to renal artery stenosis. Increased sympathetic nerve activity is associated with hypertension. Inreased sympathetic nerve stimulation, which increases renin release by the kidneys and therefore formation of angiotensin II, which, in turn, stimulates the adrenal gland to secrete aldosterone. Cardiac output is increased initially due, in part, to the additional blood flow required for the extra adipose tissue. The renal-pressure natriuresis mechanism is impaired, and the kidneys will not excrete adequate amounts of salt and water unless the arterial pressure is high or unless kidney function is somehow improved. In other words, if the mean arterial pressure in the essential hypertensive person is $150 \mathrm{~mm} \mathrm{Hg}$, acute reduction of the mean arterial pressure artificially to the normal value of 100 $\mathrm{mm} \mathrm{Hg}$ (but without otherwise altering renal function except for the decreased pressure) will cause almost total anuria, and the person will retain salt and water until the pressure rises back to the elevated value of $150 \mathrm{~mm} \mathrm{Hg}$. Chronic reductions in arterial pressure with effective antihypertensive therapies, however, usually do not cause marked salt and water retention by the kidneys because these therapies also improve renal-pressure natriuresis [5]. Retantion of salt and water in the body casues oedema, lack of vigour and morbidity.

*Corresponding author: Manjusha Joshi, Electronics and Telecommunication Department, MPSTME /NMIMS Deemed to be University, Bhakti Vedant Marg, Vile Parle-w, Mumbai, India, E-mail: Manjusha.joshi@nmims.edu

Received: January 27, 2015; Accepted: March 19, 2015; Published: March 26 2015

Citation: Joshi M, Desai KD, Menon MS (2015) Correlation between Sympathtic Power and Left Ventricular Ejection Fraction in Diabetics and Hypertensives. Bioengineer \& Biomedical Sci 5: 146. doi:10.4172/2155- 9538.1000146

Copyright: (๑ 2015 Joshi M, et al. This is an open-access article distributed under the terms of the Creative Commons Attribution License, which permits unrestricted use, distribution, and reproduction in any medium, provided the original author and source are credited. 
Hypertension is typically associated with high potassium in the blood plasma.Increase in potassium ion concentration causes vasodilation. This results from the ability of potassium ions to inhibit smooth muscle contraction.

SA node firing is controlled by complex interacting hormones epinephrine and norepine-phrine thet are sreponsible for sympathetic and parasympathetic response to any stimulous to heart. The interaction between the hormones is represented in the Figure 1. Norepinephrine released from sympathetic nervous stimulation increases myocardial contractility and Acetylcholine released from parasympathetic activity dercreses myocardial contractility.

Presynaptic release of acetylcholine can inhibit sympathetic nervous stimulation thereby reducing the extent of sympathetic stiulation. The interactive effect is referred as accentuated antagonism. Increase in vagal drive induces bradycardia for a greater extent and is also accompanied by peripherral vasoconstriction resulting in increased SNS vascular drive. Adrenal medulla is innervated by presynapltic release of Nor epinephrine to reduce the extent of release of epinephrine in the blood stream. Also the $\beta_{1}$ and $\beta_{2}$ adrenoreceptors are primed to selectively respond to Norepinephrine and epinephrine. Norepinephrine is an especially powerful vasoconstrictor hormone; epinephrine is less so and in some tissues even cause mild vasodilation. Sympathetic nervous system stimulates during stress or exercise, the sympathetic nerve endings in the individual tissues release norepinephrine, which excites the heart and contracts the veins and arterioles. The complex interaction between the two hormones leads to increased stress on the heart under prevailing hypertension. The hypertensive medication controls the heart from straining.

Prolonged and/or uncontrolled hypertension may cause Left Ventricular Diastolic Dysfunction (LVDD), Left Ventricular Hypertrophy (LVH) and both may individually or in combination lead to sudden cardiac arrest or myocardial infarction.

The HRV indices like heart rate, heart rate variability, sympatho vagal balance, orthostatic stress index and the power spectral density in $\mathrm{HF}$ band $(0.04-0.15 \mathrm{~Hz})$ are found to be distinctively different in case of diabetic subjects. The HRV analysis information is available from Auto Regressive (AR) analysis of RR intervals obtained from ECG signal acquired for the duration of 3-5 minutes in supine and sitting position.

In case of hypertensives, HRV indices like the heart rate variability (SDNN) is significantly reduced, heart rate is not affected by prevailing hypertensive condition. sympathetic power is significantly higher in hypertensive subjects.

In case of diabetic and hypertensive subjects, the increase in either sympathetic power if predominance of the hypertensive condition exists or increase in parasymapthetic power if predominance of the diabetic condition exists.

It is required to detect the $\mathrm{R}$ peak and $\mathrm{RR}$ interval samples for the HRV analysis.

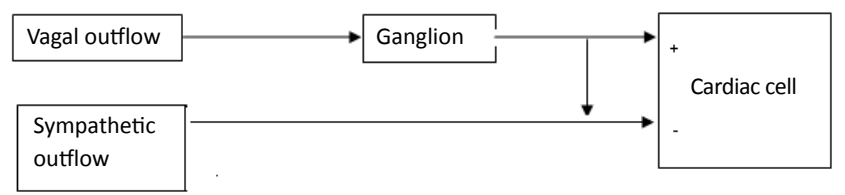

Figure 1: Interaction between vagal and sympathetic activity at pre synaptic level.

\section{QRS detector using Pan-Tompkins's algorithm}

HRV analysis requires the RR interval detalis to be extracted from ECG signal samples.So it essential to detect QRS peak from every RR interval.

When the ECG signal is acquired, it is superimposed by different types noise signals like -

1) Supply frequency interference

2) Muscle artifact

3) Baseline wander

4) T-wave interference

The above mentioned algorithm extracts the RR interval from the noisy ECG signal in the below mentioned steps.

The band pass function is realized through a design of a low pass filter and the high pass filter [3].

The low-pass filter is described by the formula-

$$
y(n)=2 y(n-1)-y(n-2)+x(n)-2(n-6)+x(n-12)
$$

and the high pass filter is described by the formula:

$$
y(n)=y(n-1)-\frac{1}{32} y(n-2)+x(n)-2(n-6)+x(n-12)
$$

The low pass filter removes the supply frequency interference (50 $\mathrm{Hz}$ ), the baseline wander which is a low frequency and the T-wave interference. The $\mathrm{T}$-wave is due to atrial repolarization that overlaps the QRS wave. The high pass filter used to remove the muscle noise interference.

The derivative filter is used to detect the QRS peak. Since it has the highest slope, the detection is possible through derivative filter. This is followed by a square filter that converts the negative spectral amplitudes to positive and also enhances the high frequency component.

$$
y(n)=\frac{1}{8}[2 x(n)+x(n-1)+x(n-3)+2 x(n-4)
$$

Moving window integration is used to incorporate the changes in the signal as the samples of the signal move ahead. The window size is directly related to the sampling rate [3]. The sampling rate of the signal is 500 samples/second and the window size 75 . The algorithm is shown in Figure 1.

$$
y(n)=\frac{1}{N}[x(n-(N-1))+x(n-(N-2))+x(n-(N-3)) \ldots x(n)]
$$

A temporal location of the QRS is marked from the rising edge of the integrated waveform. In the last step two thresholds are adjusted. The higher of the two thresholds identifies peaks of the signal. The lower threshold is used when no peak has been detected by the higher threshold in a certain time interval. In this case the algorithm has to search back in time for a lost peak. When a new peak is identified (as a local maxim mum - change of direction within a predefined time interval) then this peak is classified as a signal peak if it exceeds the high threshold (or the low threshold if we search back in time for a lost peak) or as a noise peak otherwise. In order to detect a QRS complex the integration waveform and the filtered signals are investigated and different values for the above thresholds are used. To be identified as a QRS complex, a peak must be recognized as a QRS in both integration and filtered waveform [6]. The algorithm is stated in Figure 2.

The changes in the waveform are shown step by step in Figure 3 subfigures a- $\mathrm{f}$ denote the changes due to each signal transformation. 
Citation: Joshi M, Desai KD, Menon MS (2015) Correlation between Sympathtic Power and Left Ventricular Ejection Fraction in Diabetics and Hypertensives. J Bioengineer \& Biomedical Sci 5: 146. doi:10.4172/2155- 9538.1000146

Page 3 of 6

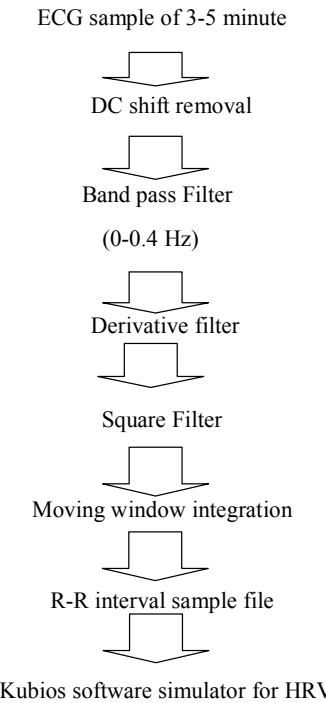

Figure 2: Pan Tompkins Algorithm.

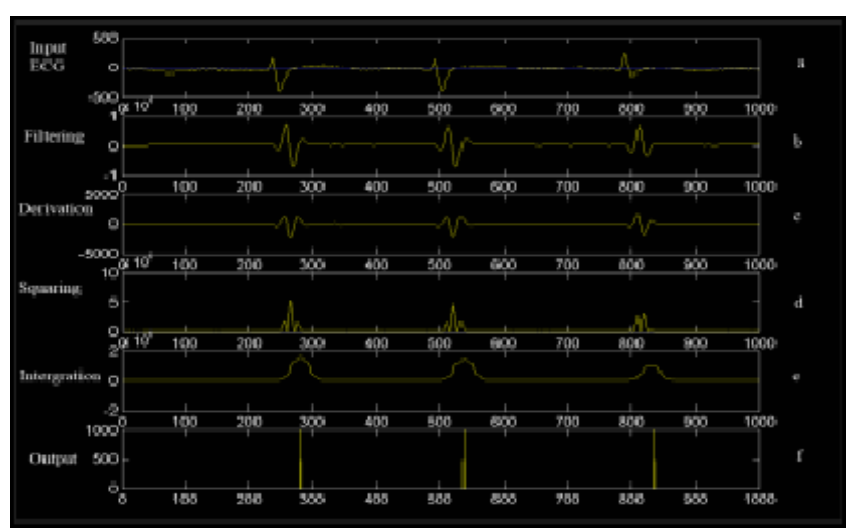

Figure 3: Denote the changes due to each signal transformation.

The $\mathrm{X}$ axis in all the waveforms from Figure 3a-3f shows the time scale. The $\mathrm{Y}$-axis is represented by voltages from Figure $3 \mathrm{a}-3 \mathrm{f}$. The Figure $3 \mathrm{f}$ wave form represents the digitized state from presence or absence of pulse. The R-R peak in figure e is observed to be matching the peak of the integrator i.e. Figure 3a-3f [6].

\section{HRV analysis tool}

The RR interval file extracted from ECG is given as an input to the HRV simulator. The Simulator is open source simulation software developed by PHYSIONET called as Kubios HRV simulator. The result sheet generated by the simulator is shown in Figure 3. The simulator lists the time domain indices, frequency domain indices and the nonlinear indices. Out of which, LF power from frequency domain analysis is analysed in the current paper.

\section{Overview of Hrv analysis}

The HRV is an old technique stated by Hales in 1956. The RR intervals of a healthy heart shows variation of greater extent compared to the impaired heart. A healthy heart is sensitive to physiological, physical and psychological changes in the body and it modifies the heart rate accordingly. It has been observed that the impaired heart has reduced the variation in the heart rate as the demanded by different activities of body. The normal person's ECG that shows more changes in the heart rate during different activities compared to that of the subject with impaired functioning of heart. The HRV analysis produces certain diagnostic indices that are obtained from spectral analysis of RR interval acquired for 3-5 minutes.

The AR analysis represents three prominent frequency bands-

1) VLF band $-0.0-0.04 \mathrm{~Hz}$

2) LF band-0.04-0.15 Hz

3) HF band- 0.15 to $0.4 \mathrm{~Hz}[5]$.

The power variations in the two bands provide a non invasive sympathetic measure of the autonomic dysfunction. Alterations due to mental stress, orthostatic stress, assessment post myocardial infarct, heart transplant surgery, evaluation of cardiac function after bypass etc can be characteristic feature [3]. Auto regressive band is used to record the data as the technique filters out unwanted frequencies.

Normalized LF power: The power in these spectra represent the baroreceptor control in LF band, sympathetic hormone controlling and inhibiting the decrease in the heart rate and parasympathetic hormone controlling and inhibiting the increase in the heart rate. Increase in parasympathetic power augments this band. Every frequency spectrum represents the normalized power spectral density from the respective band in the HRV analysis report. This power is around 50 Normalized Units (NU) in case of normal subjects, decreased in diabetic patients. The Extent of decrease is related to the prevailing cardiac performance.

Normalized HF power: The power in this band for normal subjects is around 52 Normalized Units (NU). It is found that the parasympathetic power solely dominates this band. Parasympathetic band varies with variation related to respiration around $0.25 \mathrm{~Hz}$. This error is called respiratory sinus arrhythmia. The subjects are requested to deep breath during the ECG was acquired.

The indices used in the proposed paper is - parasympathetic power derived from HRV analysis ans LVEF derived from echocardiogram. Correlation coefficient of both the indices are compared for normal, diabetic subject with and without myocardial infaction/ischemia.

Figure 4 shows the HRV report of a normal subject comparing this to Figure 5 that shows the HRV report of a diabetic subject with

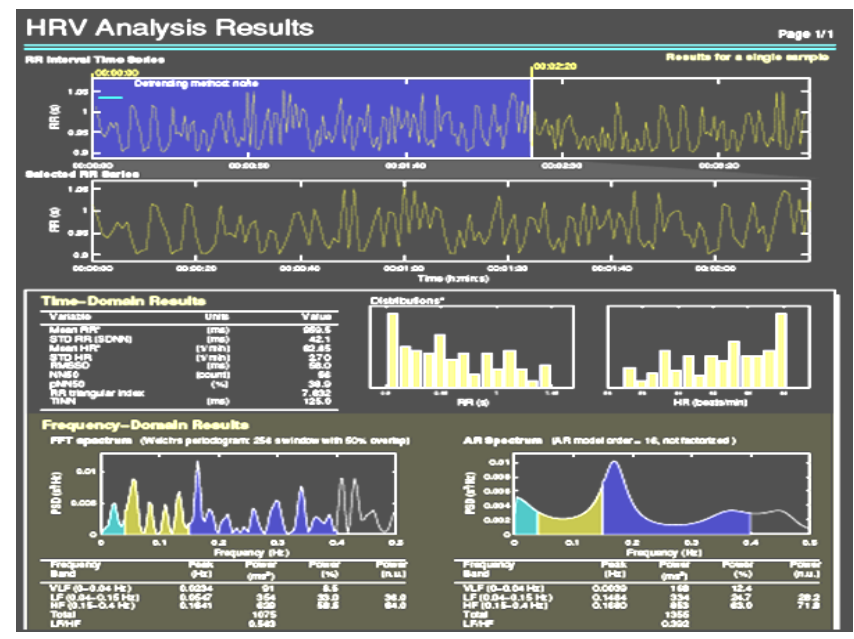

Figure 4: HRV report of a normal subject. 
Citation: Joshi M, Desai KD, Menon MS (2015) Correlation between Sympathtic Power and Left Ventricular Ejection Fraction in Diabetics and Hypertensives. J Bioengineer \& Biomedical Sci 5: 146. doi:10.4172/2155- 9538.1000146

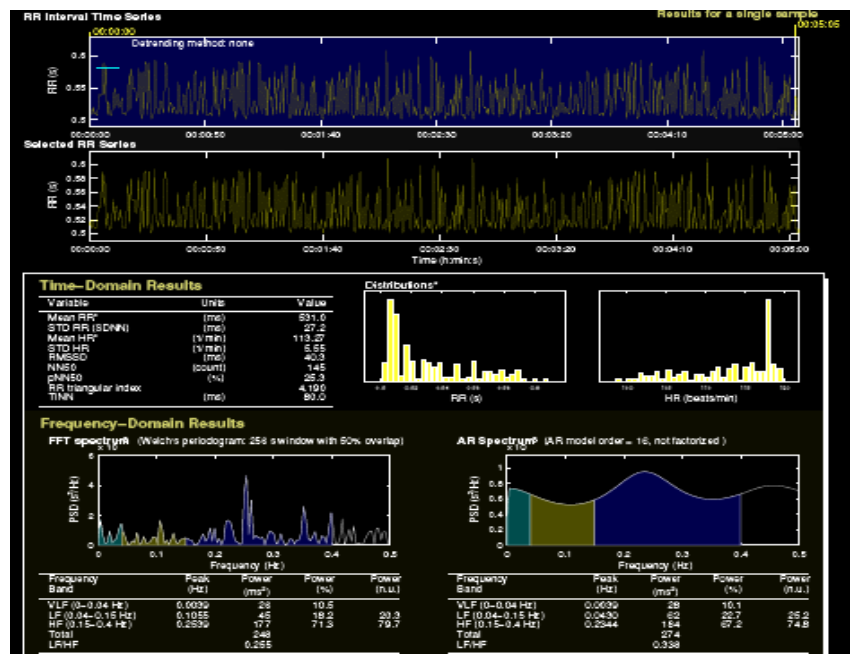

Figure 5: HRV report of a diabetic subject with myocardial infarction.

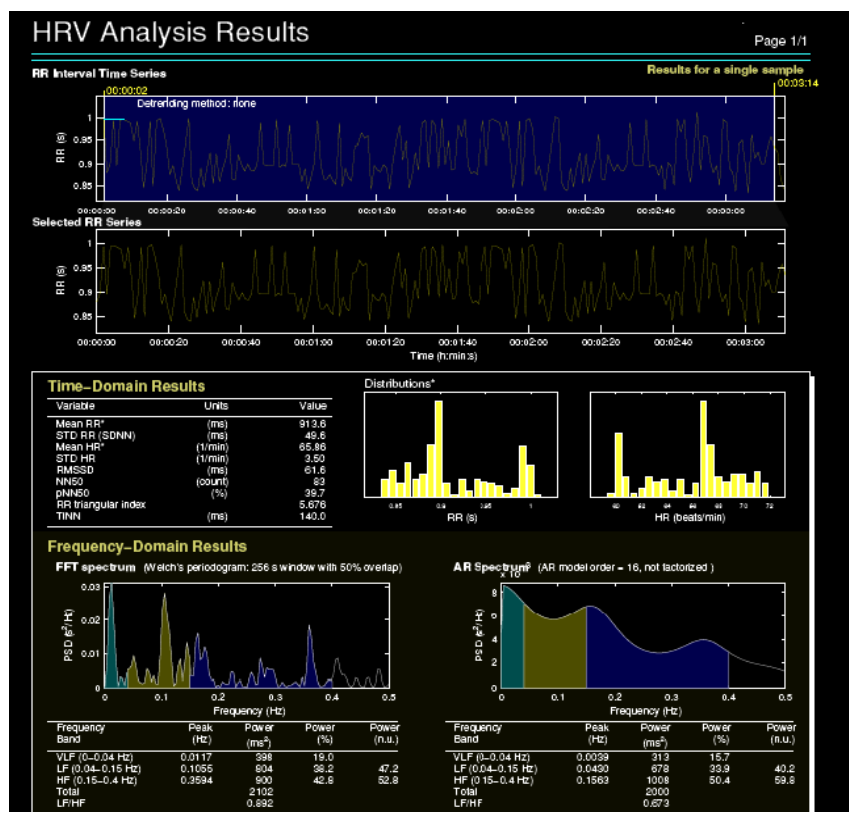

Figure 6: HRV report of a hypertensive subject.

myocardial infarction. It can be noted that heart rate is higher, heart rate variability (SDNN) is lower and sympatho vagal balance is lower than that of normal subject. The above observed indices provide confirmative diagnosis at an early stage before there is functional deterioration is clinically visible. The functional deterioration like reduced wall velocities and Left Ventricular Ejection Fraction (LVEF) can be clearly visible in echocardiogram. Figure 6 shows the increased sympathetic power in hypertensive subjects.

\section{Interpretation of echocardiogram indices}

Since the early 1950s, ultrasound use in medicine has been the basis for several procedures that are widespread in today's clinical practice. The principal application is in the field of medical imaging. Medical ultrasound imaging relies on the same principles as sonar or radar units. The ultrasound probe produces a (pulsed) acoustic pressure field. When the ultrasound signal is incident upon an elastic medium, the signal returns back without penetrating. This delay represents lowest intensity in grey scale [7]. Depending upon the density of the target velocity of the reflected signal is modified. The field propagates through the tissue and is partially reflected and scattered due to the inherent inhomogeneity of most tissues. The backscattered signal is received by the same probe and converted into a grey scale image of the organ. The probe of the ultrasound has a transmitter and a receiver in one assembly so that the organ mapping does not vary spatially. Since the ultrasound signals cannot pass from the bone, the probe is placed suitably to get suitable view. Medical ultrasound is a non ionizing radiation has several advantages over other popular imaging modalities as Magnetic Resonance Imaging (MRI), X-ray and Computed Tomography (CT). At first, unlike X-ray and CT, ultrasound is a non ionizing radiation and hence practically harmless to the human body. Computational complexity needed for the image creation is comparative less. Ultrasound systems to work at frame rates of 100 frames/sec. This makes ultrasound the standard tool for diagnosis of disease based on organs dynamics. Further advantages connected with ultrasound systems are their cost effectiveness and reduced size, making their availability possible even in small local low budget ambulatories. This is instead not the case for X-ray, CT and MRI, whose installation, besides relevant costs, requires extended dedicated areas [7]. The disadvantage of Ultrasound image is it is highly noisy [7]. Figure 3 refers to an echocardiogram showing the M-mode echocardiogram, the left ventricle can be viewed during systole and diastole. The volume change in left ventricle is directly represent the functional indicator i.e. the LVEF. LVEF can be formulated as-

$$
\mathrm{LVEF}=\frac{(\mathrm{EDV}-\mathrm{ESV})}{\mathrm{EDV}}
$$

EDV is the left ventricular end diastolic and ESV is the left ventricular end systolic volume [5]. If the heart is functioning properly, the LVEF has a higher value, It has been observed the diabetic subject suffers from vasoconstriction [4]. The lack of availability of NO due to hyperglycemia causes the vasculature in a constricted state causing vasoconstriction. As a result the body organs are deprived of nutrients. The vasoconstriction and dominance of parasympathetic activity that declines the depolarization rate of the left ventricle directly affect the proper diastolic and systolic performance. This in turn affects the contractility and the LVEF of the heart. Figure 5 shows the echocardiogram showing the left ventricular volume change during diastole and systole [5]. As can be seen from the Figure 7, LVID $_{d}$ and $\mathrm{LVID}_{\mathrm{s}}$ represent the left ventricular volume during diastole and systole

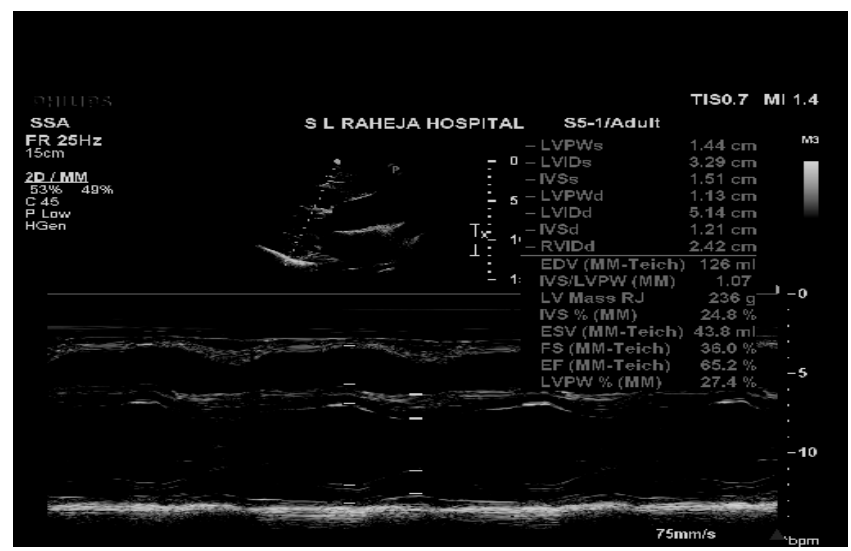

Figure 7: Echocardiogram 
Citation: Joshi M, Desai KD, Menon MS (2015) Correlation between Sympathtic Power and Left Ventricular Ejection Fraction in Diabetics and Hypertensives. J Bioengineer \& Biomedical Sci 5: 146. doi:10.4172/2155- 9538.1000146

Page 5 of 6

respectively. The contractility computed from the echocardiogram image is found to be high for normal cohort than the diabetic cohort.

\section{Data acquisition protocol, inclusion and exclusion criterion}

Study is carried out on non diabetic and non hypertensive subjects that visit the hospital for checkup or admitted in the hospital. The II LEAD ECG and echocardiogram is acquired on the same day. Data of 27 subjects of control group, 40 subjects of diabetic group with and without myocardial ischemia/infacrtion and 23 subjects hypertensive with and without diabetes are recorded.

1) All the cases were recorded from Fortis- S L Raheja Hospital.

2) Randomness in data is ensured by taking up all the cases in the stipulated period of time. The records are collected in the morning from 10.00 a.m. from 2.00 p.m.

3) All the subjects are above 25 years of age.

4) The echocardiogram and ECG acquisition equipment are same.

5) The echocardiograms from three different cardiologists are recorded.

6) The control group is non diabetic and non hypertensive subjects.

7) The diabetic subjects have at least 5 years of prevailing diabetes.

8) The clinical glycemic control record is not mandatory in diabetic cases.

9) Both male female cases are included.

The exclusion criterion states that-

1) Subjects who are younger than 25 years age.

2) Subjects suffering from type-1 diabetes.

3) Subjects with less than 5yrs. of diabetes history in study group.

4) Subjects with known history of electrolyte imbalance related ECG abnormalities.

5) Subjects with known history of Digitalis induced ECG abnormalities.

6) Subjects that are only diabetic and with no other complication are considered.

\section{Results and Discussion}

Tables $1 \mathrm{a}$ and $1 \mathrm{~b}$ show the average sympathetic (LF) power for

\begin{tabular}{|c|c|c|}
\hline LF Power & Control group & $\begin{array}{c}\text { Diabetic with a and } \\
\text { without myocardial } \\
\text { ischemia/infarction }\end{array}$ \\
\hline Mean & 36.88888889 & 51.7025 \\
\hline Variance & 73.59641026 & 5000.4771731 \\
\hline Observations & 27 & 40 \\
\hline Hypothesized mean differ & 0 & \\
\hline df & 54 & \\
\hline $\mathrm{t}$-start & -3.794905032 & \\
\hline $\mathrm{P}(\mathrm{T}<=\mathrm{t})$ one-tail & 0.000187713 & \\
\hline $\mathrm{t}$ critical one-tail & 1.673564907 & \\
\hline $\mathrm{P}(\mathrm{T}<=\mathrm{t})$ two-tail & 0.000375425 & \\
\hline $\mathrm{t}$ critical two-tail & 2.004879275 & \\
\hline
\end{tabular}

t-Test: Two-Sample Assuming Unequal Variances

Table 1a: Show the average sympathetic (LF) power for the different cohorts.

\begin{tabular}{|c|c|c|}
\hline LF Power & Control group & $\begin{array}{c}\text { Hypertensive with and } \\
\text { without Diabetes }\end{array}$ \\
\hline Mean & 36.88888889 & 31.77391304 \\
\hline Variance & 73.59641026 & 81.3529249 \\
\hline Observations & 27 & 23 \\
\hline Hypothesized mean & 0 & \\
\hline$d f$ & 46 & \\
\hline t-start & 2.043885947 & \\
\hline $\mathrm{P}(\mathrm{T}<=\mathrm{t})$ one-tail & 0.023357438 & \\
\hline $\mathrm{t}$ critical one-tail & 1.678660414 & \\
\hline $\mathrm{P}(\mathrm{T}<=\mathrm{t})$ two-tail & 0.0467144876 & \\
\hline $\mathrm{t}$ critical two-tail & 2.004879275 & \\
\hline
\end{tabular}

t-Test: Two-Sample Assuming Unequal Variances

Table 1b: Show the average sympathetic (LF) power for the different cohorts.

\begin{tabular}{|c|c|c|}
\hline LF Power & Control group & $\begin{array}{c}\text { Diabetic with a and } \\
\text { without myocardial } \\
\text { ischemia/infarction }\end{array}$ \\
\hline Mean & 0.664777778 & 0.52775 \\
\hline Variance & 0.002735564 & 0.024151218 \\
\hline Observations & 27 & 40 \\
\hline Hypothesized mean & 0 & \\
\hline df & 51 & \\
\hline $\mathrm{t}$-start & 5.160407347 & \\
\hline $\mathrm{P}(\mathrm{T}<=\mathrm{t})$ one-tail & $2.0423 \mathrm{E}-06$ & \\
\hline $\mathrm{t}$ critical one-tail & 1.675284951 & \\
\hline $\mathrm{P}(\mathrm{T}<=\mathrm{t})$ two-tail & $4.0846 \mathrm{E}-06$ & \\
\hline $\mathrm{t}$ critical two-tail & 2.007583728 & \\
\hline
\end{tabular}

t-Test: Two-Sample Assuming Unequal Variances

Table 2a: Show the average value of LVEF for different cohorts.

\begin{tabular}{|c|c|c|}
\hline LF Power & Control group & $\begin{array}{c}\text { Hypertensive with and } \\
\text { without Diabetes }\end{array}$ \\
\hline Mean & 0.664777778 & 0.643478261 \\
\hline Variance & 0.002735564 & 0.003941897 \\
\hline Observations & 27 & 23 \\
\hline Hypothesized Mean Differ & 0 & \\
\hline$d f$ & 43 & \\
\hline t-start & 1.289804771 & \\
\hline $\mathrm{P}(\mathrm{T}<=\mathrm{t})$ one-tail & 0.102006249 & \\
\hline $\mathrm{t}$ critical one-tail & 1.681070704 & \\
\hline $\mathrm{P}(\mathrm{T}<=\mathrm{t})$ two-tail & 0.204012499 & \\
\hline $\mathrm{t}$ critical two-tail & 2.016692173 & \\
\hline
\end{tabular}

t-Test: Two-Sample Assuming Unequal Variances

Table 2b: Show the average value of LVEF for different cohorts.

the different cohorts and Tables $2 \mathrm{a}$ and $2 \mathrm{~b}$ show the average value of LVEF for different cohorts. The results match the pathophysiology of the diabetic and hypertensive subjects. It can be noted that there is decrease in LF power for diabetic subjects with myocardial ischemia/ infarction as expected. The prolonged diabetic condition and poor glycemic index develops into myocardial ischemia/infaction and also consistently, the sympathetic power in this cohort is minimum. Hypertensive subjects with and without diabetes may have increased average symapthetic power indicating predominent hypertensive condition. If the subject is with prevailance of diabetic condition, sympathetic power is in observed to be reduced. The study attempts at finding out the correlation between the performance outcome i.e. LVEF and the pathophysiological reason for it.

Table 1 shows the p-value for t-test sympathetic power and Table 2 shows the same for LVEF. The critical values of one tailed test 
Citation: Joshi M, Desai KD, Menon MS (2015) Correlation between Sympathtic Power and Left Ventricular Ejection Fraction in Diabetics and Hypertensives. J Bioengineer \& Biomedical Sci 5: 146. doi:10.4172/2155- 9538.1000146

Page 6 of 6

\begin{tabular}{|c|c|c|}
\hline Type of chorot & Correlation coffecient & Absolute critical value for $\mathbf{\alpha = 0 . 0 5}$ \\
\hline Normal & 0.4093804 & 0.381 \\
\hline Diabetic with myocardial ischemia/infarction & 0.6624313 & 0.304 \\
\hline Hypertensive with a and without Diabetes & 0.44051334 & 0.413 \\
\hline
\end{tabular}

Table 3: The correlation between the sympathetic power and LVEF betweenthe control group, dibetic cohorts with and without myocardial ischemia/infacrtion and hypertensive with and without diabetes.

of unequal variance between normal and diabetic cohort with and without hypertension are tabulated. It can be observed that p-value is much below the critical value. The t-test results show the data for the afore mentioned parameters in the three cohorts is not interdependent on each other.

Table 3 shows the correlation between the sympathetic power and LVEF between the control group, dibetic cohorts with and without myocardial ischemia/infacrtion and hypertensive with and without diabetes. The correlation indicates that there is some broad class of relationship between the two different variables. The pearson's coefficient gives the degree of linearity in the relationship between the two variables [8]. The below computed relationship shows the pearson's coefficient. The values of correlation range between +1 and -1 . Positive values indicate positive correlation and negative values represent negative one. Any value below and upto 0.3 indicate that there is no correlation. Value above 0.3 suggests a medium degree of correlation. Any value equal and above 0.5 and upto 1 indicates strog relationship [8]. It can be concluded from the Table 2a, sympathetic power HF power has a medium positive correlation with LVEF for normal cohort whereas, medium negative correlation is evident from diabetic cohort diabetic with and without myocardial ischemia/infaction cohort .

The medium degree of correlation exhibits loose coupling between the sympathetic power and LVEF indicating that the LVEF is controlled by symapthetic power upto some extent and there are other controlling parameters also.

Negative correlation in normal subjects indicates that LVEF increases with decrease in sympathetic power. This is pathophysiologically consistent because in case of normal cohorts, in supine position, the cardiac performance is predominently a function of parasympathetic activity. In case of diabetic subjects, the negative correlation is found to exist but the correlation index is higher than normal cohorts. The results are pathophysiological consistent because vagal activity is more predominent in case of diabetic subjects.

Positive correlation between hypertensive with and without diabetic cohort indicates that LVEF increases with increase in sympathetic power. This is in consistence with the pathophysiology due to the fact that in case of hypertensive subjects sympathetic neurohumoral balence is skewed such that sympathetic power predominates even in supine position. The positive correlation in the hypertensive cohort with and without diabetes, indicates that hypertensive skew of neurohumoral axis. The critical coefficeints indicate the minimum value of correlation coefficient for the given number of subjects for the accuaracy of $0.05 \%$ [9]. The computed coefficients are higher than the critical correlation coefficients indicated in the Table 3 . The results may be specific to the cohorts under study and may or may not be repeated owing to the complexity of interplay of sympathetic and parasympathetic signal power (Table 3).

\section{Conclusion}

HRV index-sympathetic power and echocardiographic indexLVEF is found to have a negative medium degree of corrlation in normal subjects, a negative higher degree of corrlation in diabetic subjects with and without myocardial ischemia/infarction and a positive medium degree of correlation in case of hypertensive subjects with and without diabetes. $\mathrm{R}^{2}$ test result is not signifiant showing that the sympathetic power does not predict echocardiogram index LVEF.

\section{Further Scope}

Above findings are highly significant for the validation of HRV parameters. The same correlation can be checked for different cohorts of diabetic complication like diabetes with recorded evidence of different cardiac diseases. The same experiment can be tried for large number of cohorts on global basis.

\section{Author's Contribultion}

Dr.K.D.Desai has developed the intial concept and the details of the research to be conducted. Dr.M.S.Menon has identified the inclusion and excusion criterion for data to be collected for the studies. Manjusha Joshi has worked on the concept of the paper and developed the contests of the paper. She has developed the techical content of the paper based on physiological know how of Dr. M.S.Menon and guidence of Dr. K.D.Desai.

\section{References}

1. http://www.emri.in/images/stories/world_heart_day_2010.pdf

2. Sharma K (2013) Burden of non communicable diseases in India: Setting priority for action. Int J Med Sci Public Health 2: 7-11.

3. Arthur C, Guyton, Jackson (2006) The textbook of Medial Physiology. Elsevier Saunders pennsylvania.

4. Pitocco D, Infusino F, Zaccardi F, Scavone G, Coviello I, et al. (2010) Low glucose blood levels are associated with abnormal cardiac sympatho-vagal balance in type 2 Diabetic patients with coronary artery disease. Eur Rev Med Pharmacol Sci 14: 203-207.

5. Statistical Methods for Analysis and Processing of Medical Ultrasound Applications to Segmentation and Restoration Alma mater studiorum -università di bologna phd course: information technology xxiii cyclescientific disciplinary sector ing-inf/01,Martino Alessandrini,supervisor Prof Guidomasetti, coordinator prof. Claudio fiegna arces - advanced research center on electronic systems January 2008 - December 2010.

6. Christos Pavlatos, Alexandros Dimopoulos, Manis G, Papakonstantinou G Hardware implementation Pan Tompkins QRS detection algorithm, National Technical University of Athens. Department of Electrical and Computer Engineering Zografou 15773, Athens Greece.

7. Joshi M, Desai KD (2011) Comparative analysis of diagnostic modalities for detection of heart disease. International Journal of sunrise technologies.

8. Onlinestatbook.com/2/describing_bivariate_data/pearson.html.

9. http://www.gifted.uconn.edu/siegle/research/correlation/alphaleve.htm 